\title{
CAR T cells - what have we learnt?
}

\section{C6}

We should be cognizant of these stumbling blocks and strive to learn from our past experiences.
As we enter a new year, we look back and take stock, and in doing so one cannot help noticing the tendency of history to repeat itself. In 2017, we witnessed the first approvals relating to a ground-breaking new form of immunotherapy: chimeric antigen receptor (CAR) T cells. Fittingly, this issue of Nature Reviews Clinical Oncology features not one, but four articles related to CAR-T-cell therapy. These articles serve to remind us of the recurring challenges that seem to follow every major new development in anticancer therapy. We should be cognizant of these stumbling blocks and strive to learn from our past experiences. Indeed, the articles emphasize the need to not only anticipate acquired resistance in order to outwit this devastating disease - a seemingly insurmountable task in its own right - but also to focus our efforts on addressing the persistent, and arguably increasing, accessibility and cost barriers.

Undoubtedly, CAR T cells are another milestone in the shifting landscape of anticancer therapy. On $30^{\text {th }}$ August 2017, the anti-CD19 T-cell therapy tisagenlecleucel was approved by the FDA for the treatment of children and young adult patients with relapsed and/or refractory B-cell acute lymphoblastic leukaemia based on a remarkable overall remission rate of $82.5 \%$. As summarized in a Review by Jennifer Brudno and James Kochenderfer, such agents have also shown considerable promise in patients with B-cell lymphomas. In fact, within 2 months of the approval of tisagenlecleucel, a second anti-CD19 CAR-T-cell therapy, axicabtagene ciloleucel, was approved for the treatment of adult patients with relapsed and/or refractory large B-cell lymphoma, based on an objective response rate of $72 \%$. Nevertheless, other findings underscore that CAR T cells are by no means a panacea, with many of the usual concerns resurfacing.

Novel and potentially life-threatening toxicities are intimately associated with CAR-T-cell therapy, most commonly cytokine-release syndrome, but also encephalopathy and haemophagocytic lymphohistiocytosis. In comparison with the mostly delayed-onset, autoimmune adverse events associated with immune-checkpoint inhibitors, the toxicities of CAR-T-cells tend to be acute, less diverse, and thus more predictable. Importantly, the experience and knowledge of these toxicities is growing rapidly - maybe we are learning to expect and better delineate the characteristic toxicities of new therapies. Importantly, in a second Review, Neelapu et al. provide guidance for the management of the often severe adverse effects of CAR T cells, at a time when these agents are entering routine clinical practice.

Or are they? In a News \& Views article, Vinay Prasad considers the implications of the tisagenlecleucel approval for health-care systems. Considering that initially only a very small number of specialized cancer centres in the USA are able to offer the therapy, the likely limited access of patients to this treatment is among the multiple important factors discussed. Manufacturing issues and the hefty price tag of nearly half a million US dollars for a single infusion are also highlighted as barriers to universal access. Indeed, attention is placed on the debate surrounding the price of tisagenlecleucel, bearing in mind the substantial amount of taxpayers' money invested in the development of this agent, and that the administration of CAR T cells is a highly involved and risky procedure that requires substantial additional expenditures in the clinic, well beyond the manufacturing of the cell product itself (referred to as the "tip of the iceberg" by Prasad). The fact that most patients are unlikely to be cured makes the price tag even more contentious. Notably, $\sim 50 \%$ of patients who responded to tisagenlecleucel in the ELIANA trial relapsed within 1 year.

In this regard, maybe we are taking lessons from the past: loss of the target antigen on tumour cells is a predominant mechanism of resistance to anti-CD19 CAR T cells, and was anticipated by researchers who are already developing CARs that target different or multiple antigens, in addition to other innovative strategies to overcome the T-cell intrinsic and extrinsic restrictions of CAR-T-cell activity. Indeed, in a Research Highlight, we summarize a recent clinical study in which anti-CD22 CAR T cells showed promise in overcoming resistance to anti-CD19 therapy. Once again, however, resistance was observed.

Together, these articles illustrate both the potential and the challenges of CAR-T-cell therapy, as well as the fundamental issues that continue to plague the field of clinical oncology. Various indications suggest that we are learning to more rapidly address the biological challenges, as might be expected as we develop powerful new technologies to better understand the disease biology. Whether we are getting to grips with the wider socioeconomic issues remains less clear. What is clear is that meaningful advances in all of these areas are needed if we are to improve the outcomes of patients with cancer. 